\title{
Novel Conserved Genotypes Correspond to Antibiotic Resistance Phenotypes of E. coli Clinical Isolates
}

\author{
Michelle C. Swick ${ }^{1,2 \alpha}$, Michael A. Evangelista ${ }^{2,39}$, Truston J. Bodine ${ }^{2,49}$, Jeremy R. Easton-Marks ${ }^{5,6}$, \\ Patrick Barth $^{3,7}$, Minita J. Shah ${ }^{8}$, Christina A. Bormann Chung ${ }^{9}$, Sarah Stanley ${ }^{8}$, Stephen F. McLaughlin ${ }^{8}$, \\ Clarence C. Lee ${ }^{8}$, Vrunda Sheth ${ }^{8}$, Quynh Doan ${ }^{9}$, Richard J. Hamill ${ }^{2,4,10}$, David Steffen ${ }^{5,6}$, \\ Lauren B. Becnel ${ }^{5,6,10,11}$, Richard Sucgang ${ }^{3 *}$, Lynn Zechiedrich ${ }^{1,2,3,4,5,7 *}$
}

1 Interdepartmental Program in Cell and Molecular Biology, Baylor College of Medicine, Houston, Texas, United States of America, 2 Department of Molecular Virology and Microbiology, Baylor College of Medicine, Houston, Texas, United States of America, 3 Verna and Marrs McLean Department of Biochemistry and Molecular Biology, Houston, Texas, United States of America, 4 Interdepartmental Program in Translational Biology and Molecular Medicine, Baylor College of Medicine, Houston, Texas, United States of America, $\mathbf{5}$ Dan L. Duncan Cancer Center, Baylor College of Medicine, Houston, Texas, United States of America, 6 Biomedical Informatics Group, Baylor College of Medicine, Houston, Texas, United States of America, 7 Department of Pharmacology, Baylor College of Medicine, Houston, Texas, United States of America, 8 Life Technologies, Beverly, Massachusetts, United States of America, 9 Life Technologies, Foster City, California, United States of America, 10 Department of Medicine, Baylor College of Medicine, Houston, Texas, United States of America, 11 Department of Molecular and Cellular Biology, Baylor College of Medicine, Houston, Texas, United States of America

\begin{abstract}
Current efforts to understand antibiotic resistance on the whole genome scale tend to focus on known genes even as high throughput sequencing strategies uncover novel mechanisms. To identify genomic variations associated with antibiotic resistance, we employed a modified genome-wide association study; we sequenced genomic DNA from pools of $E$. coli clinical isolates with similar antibiotic resistance phenotypes using SOLiD technology to uncover single nucleotide polymorphisms (SNPs) unanimously conserved in each pool. The multidrug-resistant pools were genotypically similar to SMS-3-5, a previously sequenced multidrug-resistant isolate from a polluted environment. The similarity was evenly spread across the entire genome and not limited to plasmid or pathogenicity island loci. Among the pools of clinical isolates, genomic variation was concentrated adjacent to previously reported inversion and duplication differences between the SMS-3-5 isolate and the drug-susceptible laboratory strain, DH10B. SNPs that result in non-synonymous changes in gyrA (encoding the well-known S83L allele associated with fluoroquinolone resistance), mutM, ligB, and recG were unanimously conserved in every fluoroquinolone-resistant pool. Alleles of the latter three genes are tightly linked among most sequenced $E$. coli genomes, and had not been implicated in antibiotic resistance previously. The changes in these genes map to amino acid positions in alpha helices that are involved in DNA binding. Plasmid-encoded complementation of null strains with either allelic variant of mutM or ligB resulted in variable responses to ultraviolet light or hydrogen peroxide treatment as markers of induced DNA damage, indicating their importance in DNA metabolism and revealing a potential mechanism for fluoroquinolone resistance. Our approach uncovered evidence that additional DNA binding enzymes may contribute to fluoroquinolone resistance and further implicate environmental bacteria as a reservoir for antibiotic resistance.
\end{abstract}

Citation: Swick MC, Evangelista MA, Bodine TJ, Easton-Marks JR, Barth P, et al. (2013) Novel Conserved Genotypes Correspond to Antibiotic Resistance Phenotypes of E. coli Clinical Isolates. PLoS ONE 8(6): e65961. doi:10.1371/journal.pone.0065961

Editor: Willem van Schaik, University Medical Center Utrecht, The Netherlands

Received November 19, 2012; Accepted May 3, 2013; Published June 18, 2013

Copyright: (c) 2013 Swick et al. This is an open-access article distributed under the terms of the Creative Commons Attribution License, which permits unrestricted use, distribution, and reproduction in any medium, provided the original author and source are credited.

Funding: This work was supported by National Institutes of Health (NIH) RO1 Al054830 (to LZ). MCS was supported by the Pharmacoinformatics Training Program of the W. M. Keck Center for Computational and Structural Biology of the Gulf Coast Consortia (NIH grant T90 DK070109) (https://gcc.rice.edu/former_ training_programs/). TJB was supported by NIH grant T32 GM88129. No additional external funding received for this study. The funders had no role in study design, data collection and analysis, decision to publish, or preparation of the manuscript.

Competing Interests: Life Technologies, the funders of the Applied Biosystems SOLiD \$10 K Sequencing Grant, assisted in the sample processing and generation of raw data at their in-house facility. They also assisted in the initial data processing. Minita J. Shah, Christina A. Bormann Chung, Sarah Stanley, Stephen F. McLaughlin, Clarence C. Lee, Vrunda Sheth and Quynh Doan are employed by Life Technologies. Drs. Richard Sucgang, Michelle C. Swick, and Lynn Zechiedrich are co-inventors on a patent application that cover the strategy and genes uncovered in the manuscript. The Life Technologies team was interested in promoting the SOLiD technology. There are no further patents, products in development or marketed products to declare. This does not alter the authors adherence to all the PLOS ONE policies on sharing data and materials.

*E-mail: rsucgang@bcm.edu (RS); or elz@bcm.edu (LZ)

9 These authors contributed equally to this work.

a Current address: The University of Texas Health Science Center at Houston, Houston, Texas, United States of America

\section{Introduction}

Antibiotic-resistant bacterial pathogens present a grave threat to human health. Each year, approximately two million people in the United States develop bacterial infections while in the hospital [1], and more than half of these infections involve bacteria that are multidrug-resistant [1,2]. In some cases, gramnegative bacteria are resistant to nearly every existing antibiotic [2]. These hospital-acquired infections resulted in nearly 100,000 deaths in $2002[3,4]$, and are predicted to cost the 
U.S. between $\$ 5$ and $\$ 10$ billion dollars annually [5]. Multidrug-resistant bacteria contribute to increased mortality rates and lengthier hospital stays [1]. Furthermore, the cost to treat multidrug-resistant infections is $\sim 30 \%$ more than drugsusceptible infections [1].

Pathogens evolve antibiotic resistance when exposed to empirically prescribed antibiotics. Resistance mechanisms vary for each antibiotic class, and may evolve during the course of antibiotic exposure or be acquired through horizontal gene transfer. The accumulation of genetic alterations can result in a complex, polygenic phenotype (reviewed in [6]). For example, resistance to the bactericidal fluoroquinolone class of antibiotics, a popular empirical prescription choice, develops through multiple and temporally coordinated mutations and mechanisms [7-10]. Mutations in chromosomally encoded genes are the primary mechanism of resistance to fluoroquinolones. In E. coli, these mutations include the clinically relevant L83 variant of gyrA (one of two genes encoding gyrase) and I80 variant of $p a r C$ (one of two genes encoding topoisomerase IV) [7,8,11-13]. Pathogens may also acquire plasmid-encoded resistance genes such as the $q n r$ variants and $a a c-\left(6^{\prime}\right)-I b$-cr ([14-17], and reviewed in [18]). Additionally, changes in efflux pump expression can contribute to fluoroquinolone and multidrug resistance [9,19-21]. Many reports track these types of fluoroquinolone resistance mechanisms in gram-negative isolates. However, the focus on analyzing known mechanisms can occlude the discovery of novel resistance mechanisms. Some fluoroquinolone-resistant clinical isolates have fluoroquinolone minimum inhibitory concentrations (MICs) that cannot be explained by known mechanisms [20], suggesting that additional mechanisms may be present.

High throughput sequencing methods have proven exceptionally useful in identifying novel single nucleotide polymorphisms (SNPs) linked to important phenotypes. Such genomewide association studies (GWAS) are frequently employed to study genomic alterations associated with human disease [22]. Researchers have used comparative genomics to study the resistome [23,24] and have had success with clonal species like Streptococcus [25]. Although whole genome sequencing technology has become dramatically less expensive, the background of genomic variation present in large datasets presents a major computational challenge. Furthermore, given so much sequence information, the focus is still primarily on analysis of variants in genes previously implicated in resistance. We hypothesized that a pool of bacterial isolates that share an antibiotic resistance phenotype should also share a genomic signature. Sequencing genomic DNA from a pool self-normalizes, and thus dampens non-specific variations to reveal genetic variants relevant to the shared phenotype. Sequencing pools of isolates would then leverage the power of GWAS at a much lower cost.

Here, we adapted a GWAS for use with pooled genomic samples. We present evidence that the antibiotic resistance phenotype correlated with the genotype of the pool. We show that pools of multidrug-resistant $E$. coli clinical isolates with high fluoroquinolone MICs resemble the environmental isolate, SMS3-5. Our data provide support for the hypothesis that environmental bacteria may serve as a reservoir for antibiotic resistance $[26,27]$. This study uncovered three tightly linked SNPs that generate non-synonymous variants of genes encoding DNA binding proteins, which are highly conserved in fluoroquinoloneresistant E. coli.

\section{Results}

\section{Experimental Design and Sequencing Strategy}

From our curated collection of $>4,000$ clinical isolates [20,28], we selected 164 non-clonal $E$. coli, each one from a different patient, which were representative of all of the antibiotic resistance phenotypes existing in the collection. Table S1 and Table S2 show the antibiotics tested and the patient demographics for these isolates, respectively. The isolates ranged from susceptible to all tested antibiotics to multidrug-resistant and had fluoroquinolone MICs spanning six orders of magnitude [20].

We grouped the isolates into 16 pools by $k$-means clustering using Cluster 3.0 [29]. We reasoned that to best identify genetic alterations associated with antibiotic resistance, we would cluster isolates only by antibiotic resistance phenotypes and, thus, we did not include the variables of patient metadata or serotype. The parameters for the clustering algorithm included the MIC values for four fluoroquinolones and the drug susceptibility status for 17 additional antibiotics (Table S1). The number of isolates in each pool ranged from two to thirty-two. Table 1 describes the pools and their profile of drug resistance.

Because of the multiple antibiotic resistance parameters used in the clustering algorithm, only a rough ordering of the pools can be easily described. Two pools of nine isolates each were fluoroquinolone-susceptible ("S") and were also susceptible to most other antibiotics. Three pools ("H") were made up of isolates with high fluoroquinolone MICs and were resistant to at least three separate drug classes $\left(\mathrm{MDR}_{\geq 3}\right.$; [21]). The remaining pools (" $\mathrm{M}$ ") were intermediate between the other two sets, and were designated numerically (randomly) in the context of antibiotic resistance.

Besides decreasing cost, sequencing pools of isolates results in internal normalization, dampening non-specific sequence variation from individual strains while highlighting conserved genetic variants. We subjected pools of clinical isolate genomic DNA to next generation sequencing on the ABI SOLiD ${ }^{\mathrm{TM}} 3$ Platform. Mate-pair reads were mapped using 3 separate genomes (detailed below) as scaffolding platforms, and SNPs were called as described in Figure S1. SNP calls were deposited in Dryad repository (http://dx.doi.org/10.5061/dryad.r8q71). The coverage of each SNP called averaged $150 \times$. When SNPs were called as identical in nucleotide position and base identity for all reads in a pool, we refer to them as "unanimous." Unanimous SNPs had $p$-values less than $1 \times 10^{-19}$. When SNPs did not match these criteria, we refer to them as "mixed." Mixed SNPs ranged in significance, as reflected by $p$-value, because of the lack of perfect agreement within a given pool. This initial analysis considers only unanimous SNPs.

\section{Antibiotic Resistance Phenotype Correlates with Genotype on a Whole-genome Scale}

$E$. coli represents a diverse group of microorganisms with a predicted pangenome of over 16,000 homologous gene clusters. Only about 1,700 "core genes" are present in all 186 genomes sequenced to date [30]. Although next generation sequencing methods produce relatively short sequence reads, there is sufficient complexity such that calling differences from a reference genome sequence is a standard analysis technique. In most mathematical models, the sampled genome is presumably a descendant or at least closely related to the reference genome. The E. coli that made up the pools were isolated from clinics, and thus likely represent divergent evolutionary lines. Lacking a known common ancestral genome, we were confronted with the significant challenge of analyzing a potentially complex group of related genomic samples. 
Table 1. Antibiotic resistance phenotypes of pools.

\begin{tabular}{|c|c|c|c|c|c|c|c|}
\hline \multirow[t]{2}{*}{ Pool } & \multirow[t]{2}{*}{$\mathbf{n}$} & \multirow[t]{2}{*}{$\begin{array}{l}\text { MDR } \\
\text { status }\end{array}$} & \multicolumn{4}{|c|}{ FQ MIC $(\mu \mathrm{g} / \mathrm{mL})[20]$} & \multirow[t]{2}{*}{ Consensus resistance phenotype } \\
\hline & & & CIP & GAT & LVX & NOR & \\
\hline S01 & 9 & No & 0.01 & $0.01-0.02$ & $0.02-0.07$ & $0.03-0.09$ & AMP, SXT \\
\hline S02 & 9 & No & $0.01-0.09$ & $0.01-0.09$ & $0.03-0.17$ & $0.04-0.34$ & None \\
\hline M01 & 10 & $\mathrm{MDR}_{\geq 3}$ & $30-100$ & $10-30$ & $20-50$ & $200-700$ & FQs, AMP, FOX \\
\hline M02 & 10 & $M D R_{\geq 5}$ & $100-500$ & $10-30$ & $20-50$ & $200-700$ & FQs, AMP, FOX, CFZ, GEN, SXT, TIM \\
\hline M03 & 13 & No & $10-30$ & $8-30$ & $8-30$ & $50-200$ & FQs \\
\hline M04 & 23 & No & $100-400$ & $30-100$ & $50-100$ & $200-700$ & FQs, AMP \\
\hline M05 & 16 & $\mathrm{MDR}_{\geq 5}$ & $20-50$ & $10-20$ & $30-50$ & $50-300$ & FQs, AMP, FOX, SXT, TIM \\
\hline M06 & 13 & $\mathrm{MDR}_{\geq 3}$ & $50-200$ & $10-20$ & $30-100$ & $200-400$ & FQs, AMP, GEN, SXT \\
\hline M07 & 33 & $\mathrm{MDR}_{\geq 3}$ & $10-30$ & 10 & $10-20$ & $50-200$ & FQs, AMP, SXT, AMC \\
\hline M08 & 3 & No & $50-200$ & $100-300$ & $50-200$ & $100-200$ & FQs, SXT \\
\hline M09 & 3 & $\mathrm{MDR}_{\geq 3}$ & $20-30$ & 20 & $10-20$ & $100-200$ & FQs, AMP, GEN \\
\hline M10 & 5 & $\mathrm{MDR}_{\geq 5}$ & $100-400$ & $20-100$ & $30-100$ & $50-500$ & FQs, AMP,SXT, TIM, NIT \\
\hline M11 & 5 & $\mathrm{MDR}_{\geq 3}$ & $10-100$ & $10-50$ & $20-50$ & $50-200$ & FQs, AMP, SXT, NIT \\
\hline H01 & 2 & $M D R_{\geq 5}$ & $100-300$ & $10-30$ & $10-200$ & 1000 & All tested except AMK and IPM \\
\hline $\mathrm{H} 02$ & 5 & $\mathrm{MDR}_{\geq 3}$ & $50-500$ & $10-30$ & $40-50$ & $400-1000$ & FQs, AMP, FOX \\
\hline $\mathrm{H} 03$ & 5 & $\mathrm{MDR}_{\geq 3}$ & $200-500$ & $50-200$ & 100 & $400-1000$ & FQs, GEN, AMC, NIT \\
\hline
\end{tabular}

Individual isolates were pooled by $k$-means clustering and consensus resistance phenotypes (defined as the resistance profile of a majority of the isolates in a pool) were visually confirmed. Pools labeled "S" contain strains that are susceptible to fluoroquinolones and non-multidrug-resistant (non-MDR). Pools labeled "M" contain strains that are resistant to fluoroquinolones and may be $\mathrm{MDR}_{\geq 3}$ (resistant to at least 3 separate drug classes) or MDR $\geq 5$ (resistant to at least 5 separate drug classes) [21]. Pools labeled " $\mathrm{H}$ " contain strains that are $\mathrm{MDR}_{\geq 3}$ or $\mathrm{MDR}_{\geq 5}$ and have extremely high fluoroquinolone MICs. CIP=ciprofloxacin; GAT = gatifloxacin; $\mathrm{LVX}=$ levofloxacin; $\mathrm{NOR}=$ norfloxacin; $\mathrm{AMP}=$ ampicillin; SXT = trimethoprim-sulfamethoxazole; FQs = All four fluoroquinolones; $F O X=c e f o x i t i n ; C F Z=c e f a z o l i n ; \mathrm{GEN}=$ gentamicin; $\mathrm{TIM}-$ ticarcillin-clavulanic acid; $A M C=$ amoxicillin-clavulanic acid; NIT = nitrofurantoin; $A M K=$ amikacin; IPM = imipenem.

doi:10.1371/journal.pone.0065961.t001

We chose to measure the genomic variation of the pooled samples against three published and annotated $E$. coli genomes that represented susceptible and multidrug-resistant phenotypes; over $90 \%$ of the sequence reads mapped to at least one of these reference genomes. This approach reduced the complexity of the problem, allowing detection of conserved sequences among the members of a pool that differed from each particular reference; it also enabled us to use published genomic annotations for gene identification. The $E$. coli genomes chosen for comparison varied in gene content and annotation but this variation had no bearing on the variant identification. The same short sequence reads were first mapped then annotated relative to each reference independently (Figure S1).

We selected genomes of two drug-susceptible strains and one multidrug-resistant strain. The laboratory K-12 strain DH10B [GenBank: CP000948.1] has been used for multiple genome projects [31] and provides a potential host for studying the effect of new SNPs in an isogenic strain. REL606 [GenBank: CP000819.1] derives from the B lineage [32], and is the ancestral strain of the long term evolution experiment of Lenski et al. ([33], and reviewed in [34]). We hypothesized that the genotypes from drugsusceptible pools would be more similar to these reference genomes than would the drug-resistant pools.

Mapped against the $4.6 \mathrm{Mb} \mathrm{DH} 10 \mathrm{~B}$ genome, we detected $1,135,007$ unanimous and mixed SNPs across the 16 pools. Overall, the number of unanimous SNPs detected in each pool varied, but in every case the SNPs were not uniformly distributed on the DH10B chromosome (Figure S2). The sequence data from one of the multidrug-resistant (MDR) pools of isolates with extremely high fluoroquinolone MICs ( $\mathrm{H} 01)$ diverged greatly $(>25 \%)$ from all the reference genomes, suggesting that, although they were classified metabolically as E. coli, genotypically they pushed the species boundary and represented an intriguing direction of future research. We excluded this pool from further SNP analysis.

Approximately $80 \%$ of SNPs detected in fluoroquinoloneresistant pools were in coding regions, located in $92 \%$ of the 4,357 annotated genes of DH10B (Figure S3a). Unanimous SNPs accounted for 64,773 loci. Despite the differences in numbers of isolates in the pools, the number of unanimous SNP calls in each pool did not correlate with the pool size (Figure S3b). This finding indicates that the clustering algorithm effectively put similar isolates together and demonstrates the usefulness of sequencing relatively large pools of bacterial genomes.

The environmental isolate, SMS-3-5 [GenBank: CP000970.1], is resistant to many antibiotics and has the highest MICs reported for several of these drugs [27]. The SMS-3-5 genome is diverged and larger than the K12 genome (Figure S4). We hypothesized that the genomes of multidrug-resistant clinical isolates would resemble SMS-3-5. Compared to the 5.1 Mb SMS-3-5 genome, we identified SNPs on 1,450,796 loci among the 15 remaining pools. In general, pools containing the fluoroquinolone-susceptible, non-MDR isolates exhibited more SNPs (107,395 loci in S01; 94,543 in S02) than MDR pools containing isolates with high fluoroquinolone MICs (81,403 in H03; 88,677 in M11), consistent with the hypothesis that the genomes of multidrug-resistant isolates resemble SMS-3-5.

Using the SMS-3-5 genome as the scaffold, SNPs detected in most of the pools were fairly uniformly distributed, but the pools M05, M11, H02, and H03 were clustered at specific loci (Figure 1a). A large-scale inversion [27] and duplications account for the major differences between the DH10B and SMS-3-5 
genomes (Figure 1b). The regions of high SNP variation clustered near the breakpoints of these major chromosomal alterations (highlighted regions in Figure 1a), and thus may reflect regions of genomic instability. The same large chromosomal inversion can be found, in part, in at least one other strain from E. coli phylogenetic group D, the same group as SMS-3-5 [35]. This inversion, however, is not present in any of the other phylogenetic groups (Figure S5).

The number of SNPs called is a direct measure of the difference between that pool and the reference. Thus the $\log$ ratio of the number of unanimous pool-specific SNPs relative to either DH10B or SMS-3-5 (Figure 2a) reveals the similarity of each pool to the reference genomes (Figure 2b). The genomes of the multidrug-resistant clinical isolates with high fluoroquinolone MICs in the $\mathrm{H} 02$ and $\mathrm{H} 03$ pools were more similar to the environmental isolate than to DH10B. Likewise, the genomes in the drug-susceptible pools were more similar to DH10B.

We refined the SNP analysis by comparing the number of unanimous SNPs in common between any two pools relative to every other pool. The commonality of SNPs is a similarity metric that defines a distance map among pools. This similarity can be represented as a hierarchical tree (Figure 2c). The branch length of each tree in the axes denotes their unrooted relative distance in the hierarchical clustering process. The dot plot compares the hierarchical clustering of the pools using SNPs called against $\mathrm{DH} 10 \mathrm{~B}$ or SMS-3-5. The pools that were the most drugsusceptible clustered together and pools that were the most drug-resistant clustered together, regardless of which reference genome was used, again illustrating the relative independence of the pool-based sequencing to the reference genomes. One pair of multidrug-resistant pools (M5 and M11) also clustered together, indicating genotypic similarity. The dispersed nature of the remaining pools suggests that the key genotypic differences for antibiotic resistance and susceptibility involve different genomic regions in E. coli. This idea is consistent with the differences in distribution of SNPs, which were dependent on the scaffold used (Figure 1a). Phenotypic differences between the two genomes, therefore, are not a consequence of variations in a core set of genes, such as antibiotic target or resistance-encoding genes.

About half of all the unanimous SNPs were specific to a single pool (Figure S3c). The remaining unanimous SNPs were shared between at least two pools; $1.6 \%$ of which were found in all 13 pools of fluoroquinolone-resistant pools. Among all unanimous SNPs, homotypic changes (purine to purine or pyrimidine to pyrimidine) were detected twice as frequently as heterotypic SNP conversions, in agreement with most [36,37], but not all [36] metazoan and human data. SNPs in metazoan genomes are usually biallelic (two variants per locus), attributed to the relative rarity of single nucleotide mutations and the long generation times of these organisms. Given the short generation times and active DNA metabolism of bacteria, we expected a higher rate or triallelism or even tetraallelism in polymorphic loci in our dataset. We discovered, however, that biallelic loci accounted for $99.2 \%$ of the SNPs we measured, with a scant few $(0.8 \%)$ triallelic. At no single position were all four nucleotide variants measured.

\section{Novel SNPs Linked to Fluoroquinolone Resistance Represent a Potential Resistance Fingerprint}

With the high frequency of biallelism we observed, SNPs from different phenotypic pools could be subtracted directly to reveal a potential genetic signature specific to a selected phenotype. We created a computational platform that performs arbitrary set arithmetic between the pools based on their phenotypes to enrich for alleles that are linked to a specific phenotypic character. From these sets of loci, we determined the annotated genes affected by non-synonymous changes, and established their commonality between the reference genomes to leverage the preexisting annotations. To our knowledge, this is the first time SNP subtraction has been carried out on pooled data. This approach can be generalized to enrich for SNPs linked with any phenotype for which large enough sequence data exist. As our dataset derives from a collection best studied for fluoroquinolone resistance $[20,21,28]$, we performed the subtraction designed to enrich for alleles associated with this trait.

SNPs that occurred in either of the fluoroquinolone-susceptible pools ( $\mathrm{S} 01$ and $\mathrm{S} 02$ ) were subtracted from the SNPs conserved in all 13 fluoroquinolone-resistant pools to reveal SNPs enriched for fluoroquinolone resistance (Figure 3a). Relative to the genome of DH10B, 230 unanimous SNPs in coding and noncoding regions were shared among all the fluoroquinolone-resistant pools, but were not unanimous in the fluoroquinolone-susceptible pools. Six of these SNPs were predicted to make non-synonymous changes to annotated genes (Figure 3a, genes contained within the blue circle). Against the REL606 genome, 989 SNPs conformed to the same fluoroquinolone-resistance-based criteria; 117 of these were non-synonymous (Figure 3a, genes listed within the green circle). Relative to SMS-3-5, one SNP associated with fluoroquinolone resistance was unanimous and corresponded to a non-synonymous change in the gene, EcSMS35_3015. When aligned using Gene Stream (http://xylian.igh.cnrs.fr/bin/align-guess.cgi), this gene shared $98.6 \%$ nucleotide sequence identity with the $y g f O$ gene of DH10B, which encodes a xanthine/uracil permease. This SNP may be an important distinction between the fluoroquinoloneresistant clinical isolates and the environmental isolate.

To generate a SNP signature for fluoroquinolone resistance without biasing toward any single reference genome or any single $E$. coli strain, we only considered alleles that were in agreement with SMS-3-5, but deviated from both DH10B and REL606 (Figure 3a, genes contained within the blue $(\mathrm{DH} 10 \mathrm{~B})$ and green (REL606) circles). Gene exclusivity from the Venn diagram could result from variations linked to the phenotype of the specific reference, or could be a consequence of a gene being absent from the annotation of either of the two other reference genomes. Conversely, inclusion of a gene in the Venn diagram strongly suggests some sort of involvement with fluoroquinolone resistance. We identified SNP variants of $\operatorname{gyr}$, $\operatorname{lig} B$, mutM, and $\operatorname{rec} G$ genes linked to fluoroquinolone resistance. The well-known L83 allele of gyrA exists in all of the fluoroquinolone-resistant isolates in this collection [20], and is the most common variant seen in fluoroquinolone-resistant $E$. coli and corresponding mutations in other gram-positive and gram-negative bacteria (reviewed in [38]). Identifying this gyrA allele supports the hypothesis that our subtraction method reveals SNPs associated with fluoroquinolone resistance.

The other three coding loci with SNPs discovered by this subtraction method correspond to non-synonymous variations in recG (A109T), mutM (A422S), and $\operatorname{lig} B$ (T127A), alleles that have never before been associated with fluoroquinolone resistance. Sanger sequencing confirmed the SNP variants in a subset of isolates representing all 15 pools (Table S3). For all three SNP variants, the variant was found in all resistant isolates and in several, but not all susceptible isolates. The difference in frequency with which these SNP variants were found in resistant pools compared to susceptible pools was significant by Chi-square test. Confirmation of these SNPs within individual isolates also validated that our gene annotation algorithms were unbiased toward any single reference. 
A

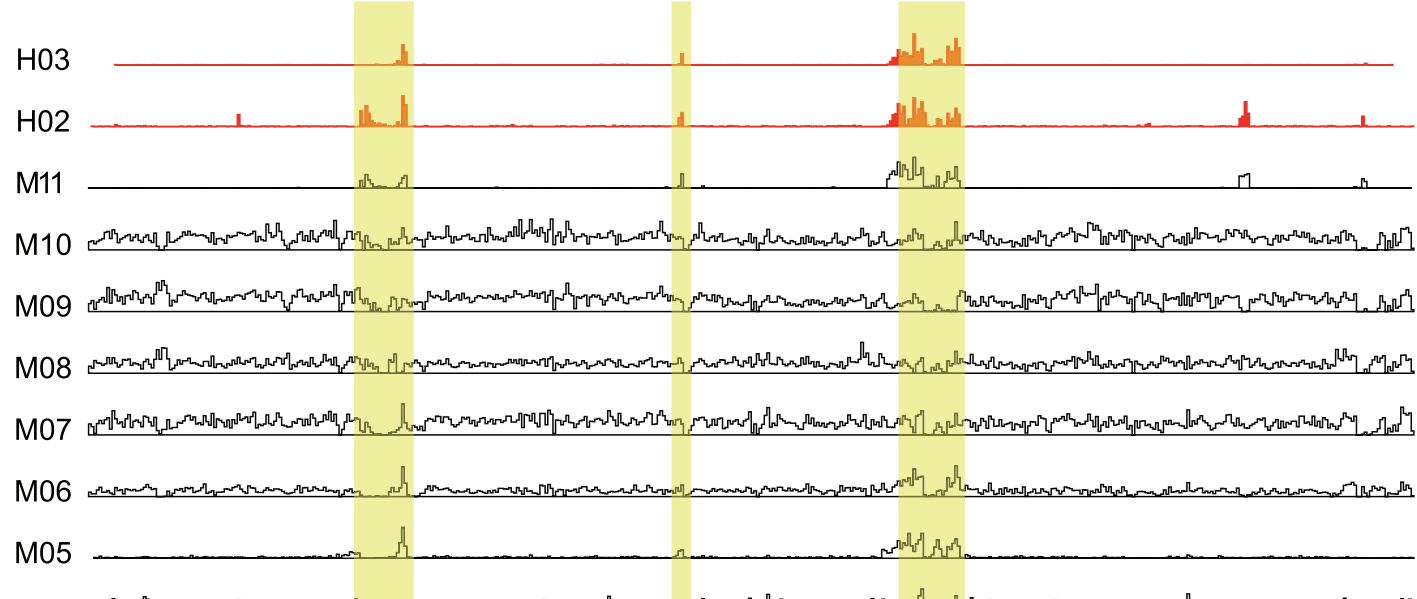

M04

M03

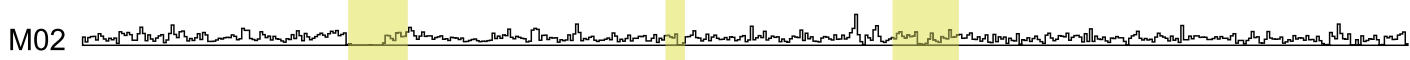

M01

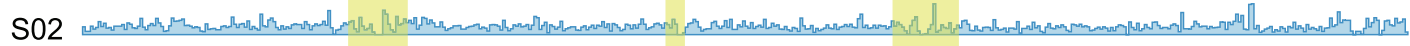

B

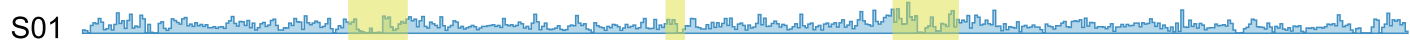

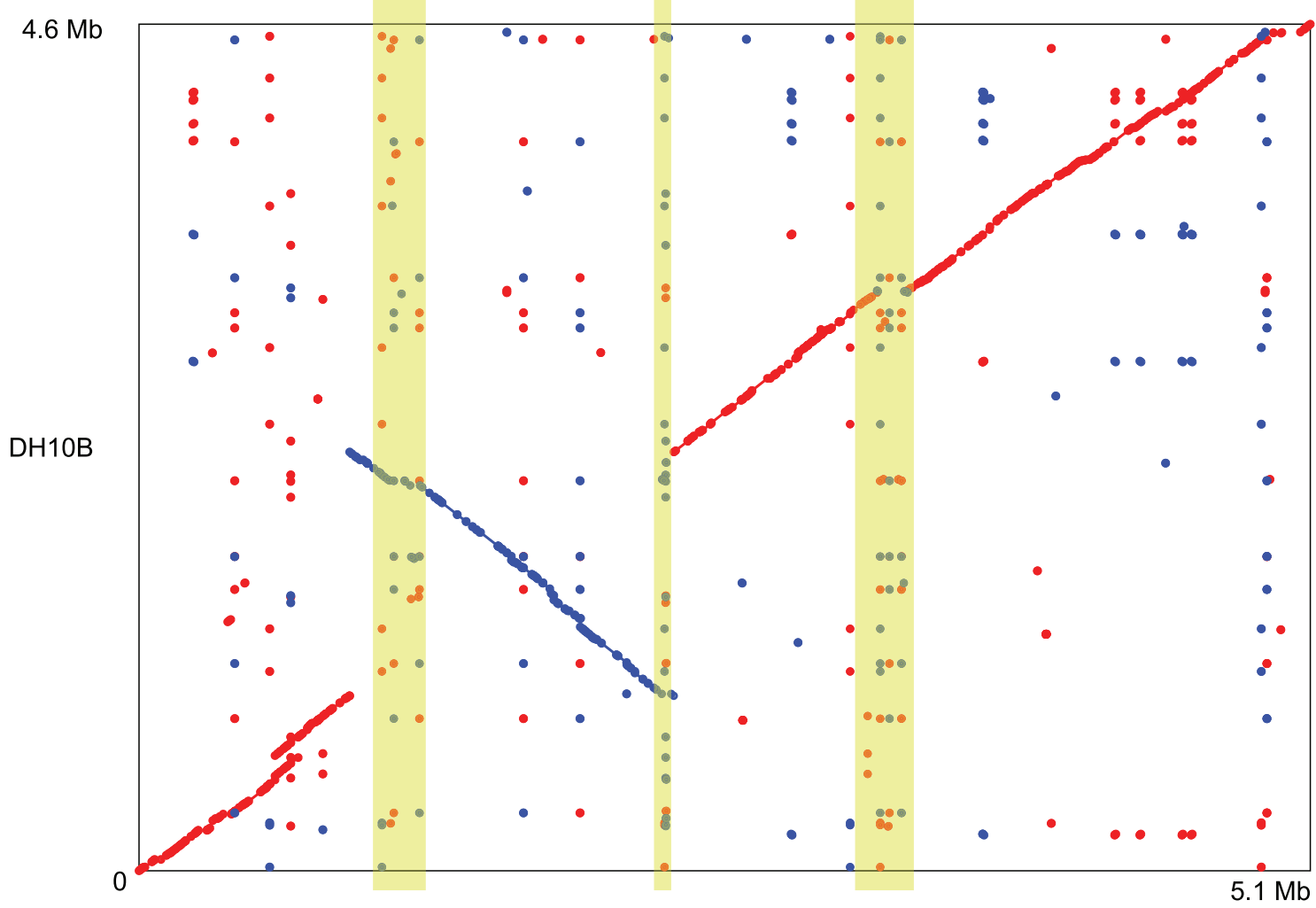

SMS-3-5

Figure 1. Pools of multidrug-resistant $E$. coli clinical isolates are genotypically similar to SMS-3-5. A) The distribution of SNPs in each pool along the chromosome was plotted against the SMS-3-5 reference genome. The $y$-axis for each pool represents a frequency of SNPs within binned regions of the chromosome. The height of the peaks was maximized to allow visualization of SNPs and does not imply a value of frequency relative to other pools. Peaks in the plot represent regions of high relative variability for a pool. " $\mathrm{S}$ " pools are shown in blue; " $\mathrm{H}$ " pools in red; " $\mathrm{M}$ " pools in black. B) Dot plot of DH10B and SMS-3-5. The DH10B and SMS-3-5 genomes were aligned using MUMmer. The SNP distribution plots for each pool were aligned to the dot plot in (A). Red dots show aligned contiguous sequences; blue dots show inversions. Highlighted in yellow are regions of consistent high SNP distribution across all pools.

doi:10.1371/journal.pone.0065961.g001 
A

\begin{tabular}{|c|c|c|}
\hline Pool & $\begin{array}{r}\text { \# of } \\
\mathrm{DH} 10 \mathrm{~B}\end{array}$ & $\begin{array}{l}\text { VPs } \\
\text { MS-3-5 }\end{array}$ \\
\hline$\overline{\mathrm{H} 02}$ & 4692 & 1878 \\
\hline $\mathrm{H} 03$ & 4460 & 1364 \\
\hline M01 & 3324 & 17660 \\
\hline M02 & 6765 & 15684 \\
\hline M03 & 1830 & 35531 \\
\hline M04 & 8799 & 11628 \\
\hline M05 & 1905 & 1929 \\
\hline M06 & 5600 & 11146 \\
\hline M07 & 1233 & 15732 \\
\hline M08 & 2594 & 23409 \\
\hline M09 & 8946 & 53659 \\
\hline M10 & 3172 & 32910 \\
\hline M11 & 2591 & 2139 \\
\hline S01 & 4606 & 12762 \\
\hline S02 & 4256 & 13286 \\
\hline
\end{tabular}

B

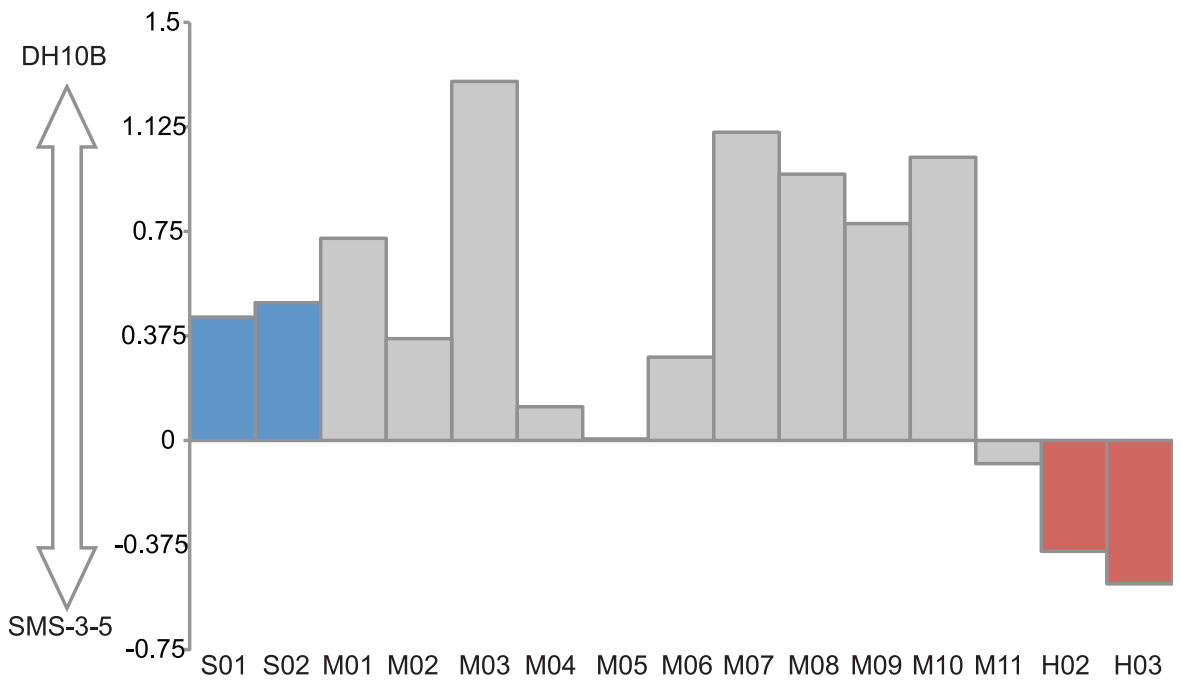

C

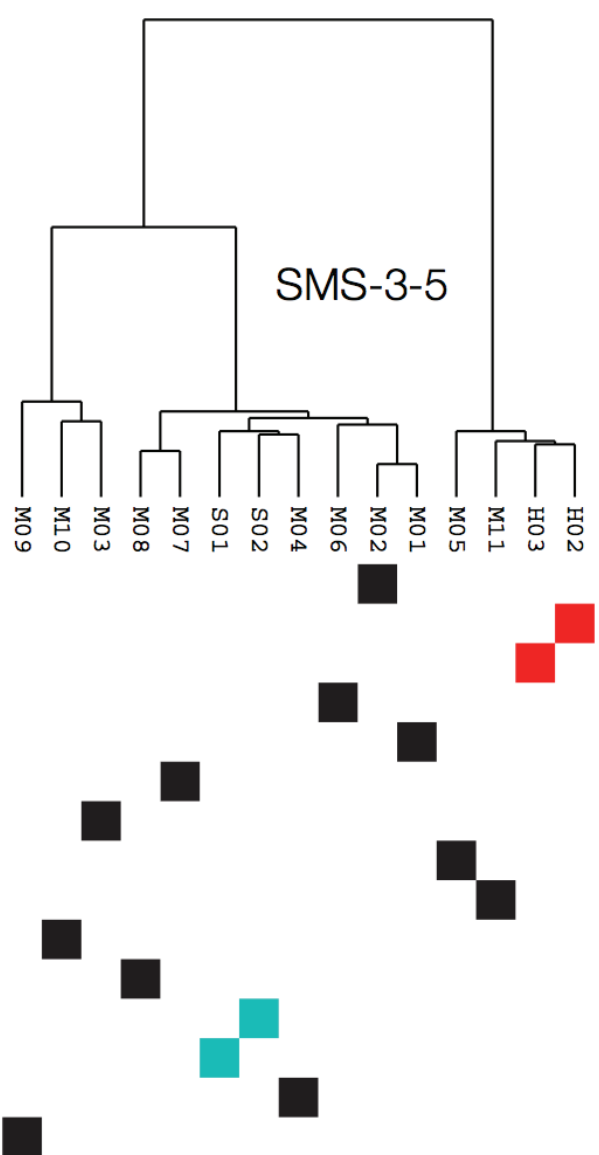

Figure 2. Relationship of pool consensus sequences to susceptible genome DH10B and resistant genome SMS-3-5. A) Total numbers of unanimous SNPs in each pool relative to DH10B and SMS-3-5. B) The similarity of each pool to DH10B and SMS-3-5. The logarithm of (unanimous SNPs relative to SMS-3-5/unanimous SNPs relative to DH10B) was plotted for each pool. Blue bars are "S" pools; red bars are " $\mathrm{H}$ " pools; gray bars are "M" pools. C) Dot plot of genomic similarity for each pool. The genomic similarity of each pool was calculated relative to each DH10B and SMS-3-5, resulting in a hierarchical clustering of the pools. Blue squares are " $\mathrm{S}$ " pools; red squares are " $\mathrm{H}$ " pools; black squares are " $\mathrm{M}$ " pools. doi:10.1371/journal.pone.0065961.g002 

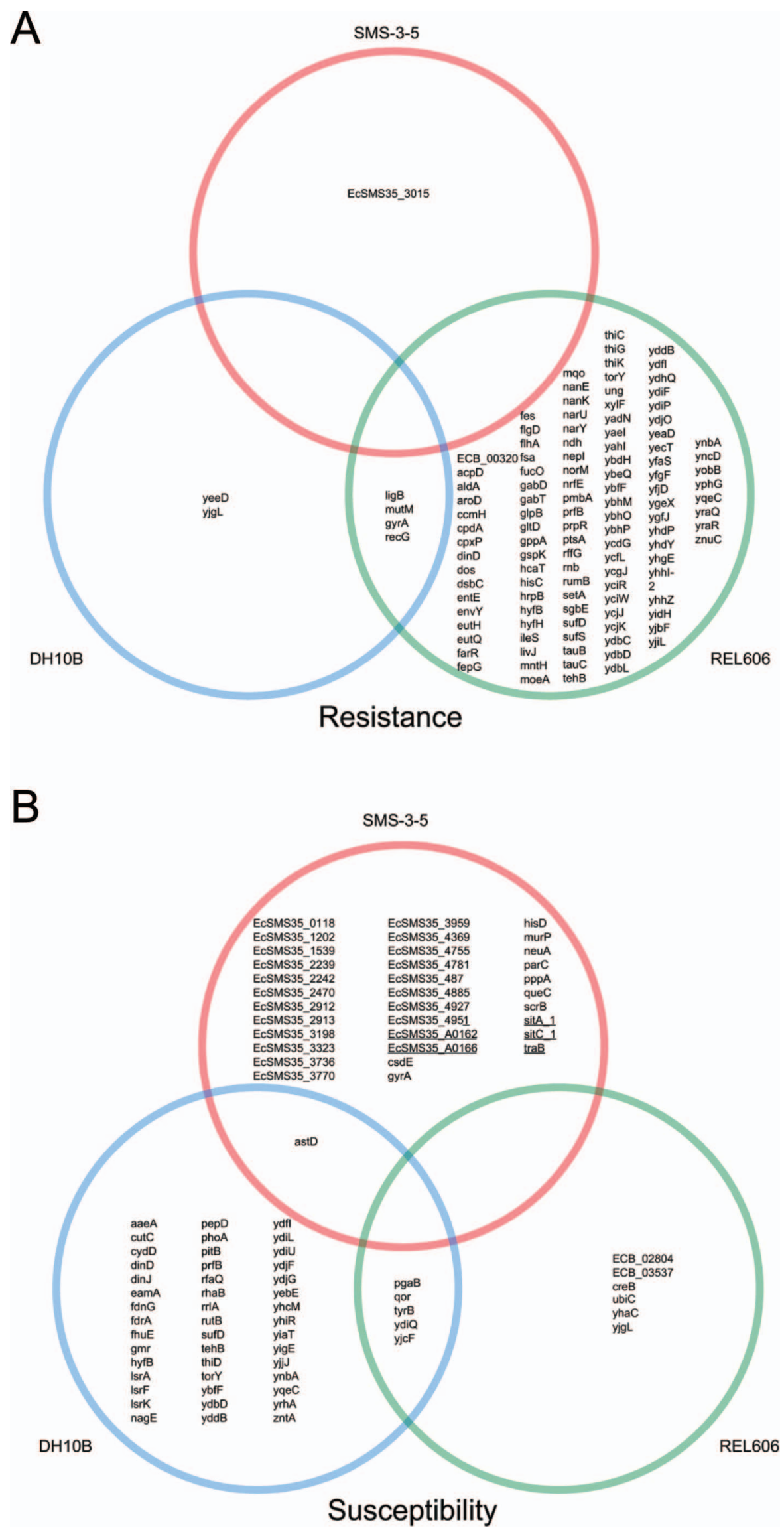
Figure 3. SNPs associated with fluoroquinolone resistance and susceptibility. Unanimous SNPs that result in non-synonymous changes in genes were computed relative to each of the three reference genomes, SMS-3-5 (multidrug-resistant with high fluoroquinolone MICs), DH10B, and REL606 (both antibiotic susceptible). Genes containing these variants for each reference genome are shown in Venn diagrams. A) Genes with allelic variants that were enriched in the fluoroquinolone-resistant phenotype. Variants that were called in any fluoroquinolone-susceptible pool were subtracted from those found in common among all fluoroquinolone-resistant pools. B) Genes with allelic variants that were enriched in the fluoroquinolone-susceptible phenotype. Underlined genes are encoded on an SMS-3-5-specific plasmid (pSMS35_130). doi:10.1371/journal.pone.0065961.g003

\section{Novel SNPs Linked to Fluoroquinolone Resistance Demonstrate a Survival Phenotype under Genotoxic Stress}

Deletion of $\operatorname{rec} G$ increases fluoroquinolone susceptibility [39] and causes replication defects [40] in E. coli. To test whether these SNP variants contribute to fluoroquinolone resistance, we used the individual null mutants for $m u t M, \operatorname{lig} B$, and $\operatorname{rec} G$ from the Keio knock-out strain collection [41]. Through sequencing, we found that the Keio parent strain matched DH10B in nucleotide identity at the $\operatorname{rec} G$, $m u t M$, and $\operatorname{lig} B$ alleles. We then measured fluoroquinolone MICs for each mutant strain. Isogenic null mutants had ciprofloxacin MICs that were $\geq 2$-fold lower than the parent (Figure 4a). For mut $M$ and $\operatorname{rec} G$, plasmid-encoded complementation restored ciprofloxacin MIGs to parent strain levels. The plasmid expressing $\operatorname{lig} B$ did not fully restore the MIC to that of the parent strain. Transformation of the parent strain with the $\operatorname{lig} B$ expression plasmid also lowered the ciprofloxacin MIC (Figure 4a).

The genes $\operatorname{lig} B$, mut $M$, and $\operatorname{rec} G$ all encode proteins known to interact with DNA, and both mutM and $\operatorname{rec} G$ are known to be involved with the repair of DNA damage. Fluoroquinolone exposure results in cellular release of reactive oxygen species (ROS) and 8-oxoguanine nucleotides, which could cause significant damage to the chromosome [42]. It has been shown that a double deletion mutant of both mutM and mut $Y$ survives fluoroquinolone exposure better than the isogenic parent strain. This phenotype was attributed to a reduction in double-strand DNA breaks [43]. Fewer breaks in the chromosome and increased mutation rate could be advantageous in the presence of stress such as from fluoroquinolones. Given the role of MutM in repair of such damage, we tested whether the variants of LigB, MutM, and RecG responded differently to ROS. Plasmids encoding either gene variant were transformed into the respective null strains, and then exposed to genotoxic stress. When exposed to $5 \mathrm{mM} \mathrm{H}_{2} \mathrm{O}_{2}$, the fluoroquinolone-resistant associated variant of MutM (A127) survived $\sim 2$ fold better than the T127 variant (Figure $4 \mathrm{~b}$ ). When exposed to ultraviolet (UV) radiation, the T127 variant survived better at low doses and the fluoroquinolone resistance-associated variant survived better at high doses (Figure 4c). The resistanceassociated variant of MutM may lower the activity or affinity for 8oxoguanine lesions in the chromosome, resulting in fewer breaks in the chromosome. The cell thus would have fewer breaks to repair than would the wild-type variant.

LigB has only a weak ligase activity in vitro [44]. If the resistanceassociated LigB variant contributes to increased ligation activity, the cell could benefit under stress. We found that the resistanceassociated LigB variant, S422, was $\sim 10$ fold more susceptible to $\mathrm{H}_{2} \mathrm{O}_{2}$, but this same allele better survived UV radiation. There was no significant difference observed between the two RecG variants for survival with either $\mathrm{H}_{2} \mathrm{O}_{2}$ or UV stress. The $\operatorname{rec} G$ allele differences may only become apparent when combined with the other alleles.

To determine where within each protein the SNP variants were located, we searched for crystal structures in the Protein DataBase (PDB; http://www.rcsb.org/pdb/home/home.do). Co-crystal atomic structures with DNA exist for three of the four proteins (GyrA PDB ID: 2XCR; MutM PDB ID: 1K82; and RecG PDB
ID: 1GM5). LigA was used to model LigB (LigA PDB ID: 2OWO). The amino acids impacted by the allelic variants are all located in alpha helices directly adjacent to a domain that interacts with DNA (Figure 5). Remarkably, the variants in $\operatorname{lig} B$, mut $M$, and rec $G$ are all located at the "cap" of these alpha helices. Thus, the variable amino acid residues may affect protein interactions with DNA.

\section{SNPs Associated with Fluoroquinolone Resistance are Linked}

The alleles of $g y r A, \operatorname{lig} B, \operatorname{mut} M$, and $\operatorname{rec} G$ were conserved in all of our tested fluoroquinolone-resistant $E$. coli clinical isolates (Table S3). To determine how frequently these variants occur in other sequenced $E$. coli, we analyzed the 39 fully annotated, complete $E$. coli genomes archived in GenBank and the 83 annotated draft genomes from the Broad Institute Escherichia coli Antibiotic Resistance Sequencing Project, Broad Institute of Harvard and MIT (http://www.broadinstitute.org/). Ten genomes contained the gyrA L83 variant; nine of these genomes also contained all three of the novel resistance-linked variants of the genes. The tenth genome encoded the $\operatorname{lig} B$ and $\operatorname{rec} G$ variants, but not the mutM variant. The gyrA L83 allele, then, is 100\% linked to the $\operatorname{lig} B$ and rec $G$ alleles and $90 \%$ linked to all three. Such tightly linked inheritance implies a linked activity that is relevant to fluoroquinolone resistance. Of 122 strains in GenBank and Broad Institute, 109 encoded either none ( 15 strains) or all three (94 isolates) of the resistance-associated alleles. Of the remaining 13 isolates and strains, four encode the resistance-linked alleles of $\operatorname{lig} B$ and $\operatorname{rec} G$, four encode the mut $M$ and $\operatorname{lig} B$ alleles, and five encode the mut $M$ allele alone.

The $\operatorname{lig} B$, mut $M$, and $\operatorname{rec} G$ genes are encoded in a $15 \mathrm{~kb}$ cluster on the chromosome (Figure 6a). Linkage of alleles among these three genes could be explained by their close chromosomal proximity through co-inheritance or from selective pressure. If the three alleles are co-inherited through physical linkage, then all of the alleles within the cluster should be linked. We identified biallelic variants: one that occurs in $\mathrm{rad} C$, encoding a DNA repair protein, located between $m u t M$ and $\operatorname{lig} B$; and spoT, encoding a (p)ppGpp synthase, located between $\operatorname{lig} B$ and $\operatorname{rec} G$ (Figure 6a). We performed pair-wise comparisons across the genomes in GenBank and the Broad Institute. Comparison of either spoT or $\operatorname{rad} C$ with $m u t M$, rec $G$, or $\operatorname{lig} B$ showed that these variants were not linked, and their probability of being found together was roughly equal to that of random chance (Figure 6b and Figure 6c). However, mut $M, r e c G$, and $\operatorname{lig} B$ were very tightly linked. This result suggests that physical proximity of the $m u t M$, $\operatorname{lig} B$, and $\operatorname{rec} G$ loci is insufficient to explain the tight linkage of the alleles. Instead, the three alleles may be under a positive evolutionary selection. For example, they might cooperatively provide an adaptability advantage during exposure to antibiotics. Alternatively, it may be that the genes in this region were once physically linked, but the intervening genes were under different selective pressure to separate away from the other genes. Selective pressure of $m u t M$, $\operatorname{lig} B$ and $\operatorname{rec} G$ linkage seems more likely given that the pair-wise linkage of the interspaced genes approximated random chance. 


\begin{tabular}{|c|c|c|c|c|c|}
\hline \multicolumn{2}{|c|}{$\Delta m u t M$} & \multicolumn{2}{|c|}{$\Delta \operatorname{lig} B$} & \multicolumn{2}{|c|}{$\Delta r e c G$} \\
\hline Strain & CIP MIC $(\mu \mathrm{g} / \mathrm{mL})$ & Strain & CIP MIC $(\mu \mathrm{g} / \mathrm{mL})$ & Strain & CIP MIC $(\mu \mathrm{g} / \mathrm{mL})$ \\
\hline$\Delta m u t M$ & 0.008 & $\Delta \operatorname{lig} B$ & 0.008 & $\Delta r e c G$ & 0.003 \\
\hline+ empty vector & 0.016 & + empty vector & 0.012 & + empty vector & 0.006 \\
\hline + MutM(T127) & 0.016 & + LigB(A422) & 0.016 & $+\operatorname{RecG}(\mathrm{A} 109)$ & 0.012 \\
\hline + MutM(A127) & 0.016 & $+\operatorname{LigB}(S 422)$ & 0.016 & $+\operatorname{RecG}(\mathrm{T} 109)$ & 0.012 \\
\hline
\end{tabular}
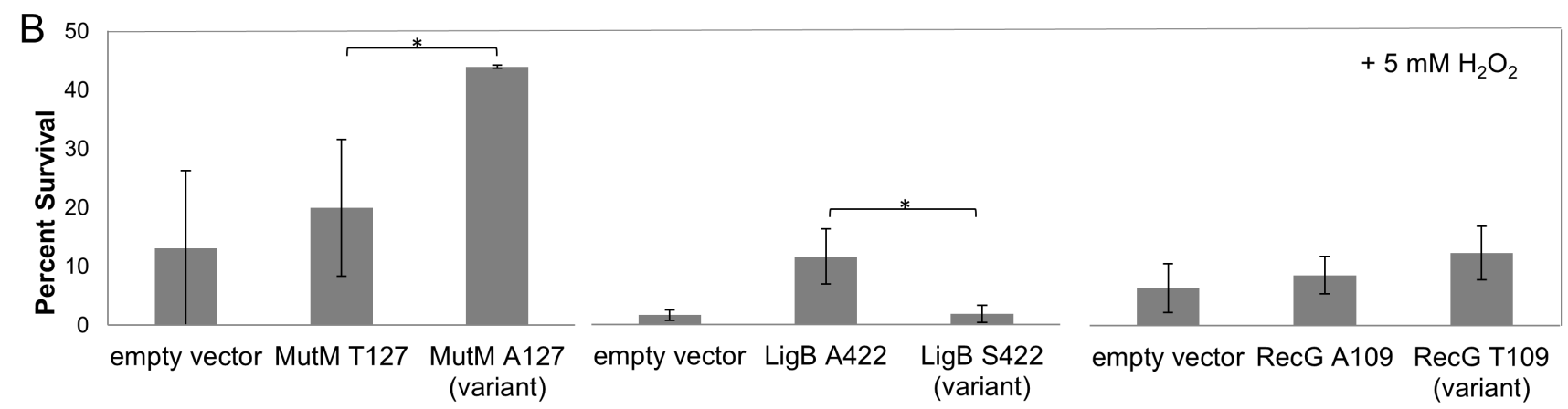

C
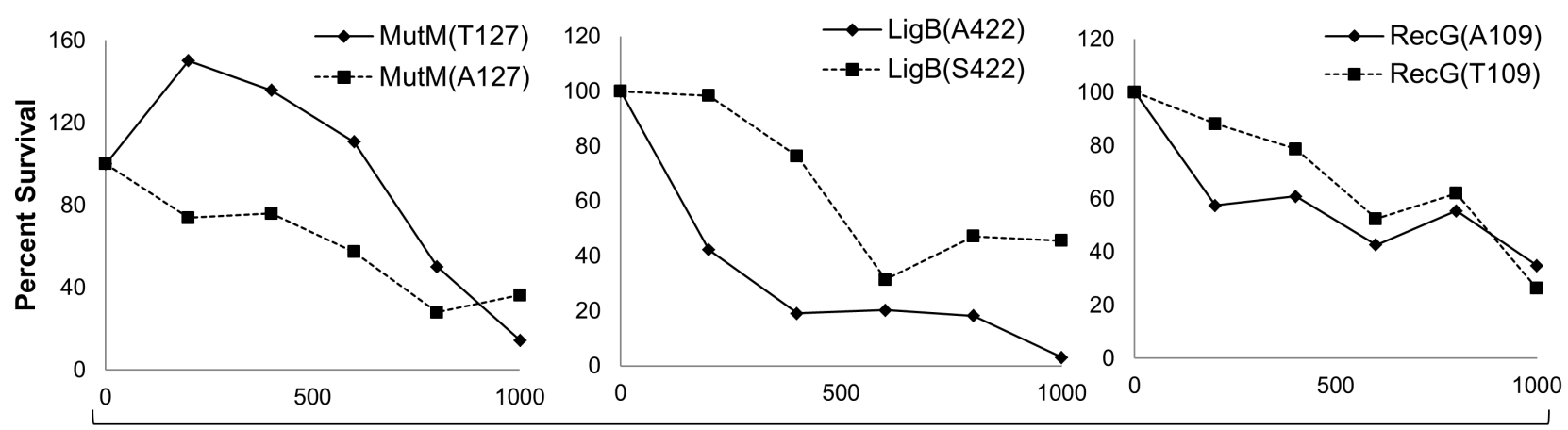

UV Dose $\left(\mu \mathrm{J} / \mathrm{cm}^{2}\right)$

Figure 4. Effect of variants on ciprofloxacin, hydrogen peroxide, or ultraviolet light susceptibility. A) Ciprofloxacin (CIP) susceptibility. MICs were measured using E-test. Experiments were repeated twice with identical results. The CIP MIC for the parent strain was $0.016 \mu \mathrm{g} / \mathrm{ml}$. B) $\mathrm{H}_{2} \mathrm{O}_{2}$ susceptibility. Cells were grown and exposed to $5 \mathrm{mM} \mathrm{H}_{2} \mathrm{O}_{2}$ for 20 minutes, then spread onto LB agar. Colonies were counted after overnight incubation at $37^{\circ} \mathrm{C}$. Shown are the averages for three independent experiments. * $p$-value of $<0.05$. C) UV susceptibility. Cells were spread onto LB agar and exposed to various doses of UV light. Shown is a representative result of three separate experiments. doi:10.1371/journal.pone.0065961.g004

\section{Novel SNP Variants Correspond to Fluoroquinolone Susceptibility}

Above, following the traditional mindset of genetic alteration associated with disease, we analyzed SNPs associated with fluoroquinolone resistance. We wondered whether there might be SNPs associated with fluoroquinolone susceptibility. To identify potential genomic signatures linked to antibiotic susceptibility, we subtracted SNPs found in any of the "M" or " $\mathrm{H}$ " pools from unanimous SNPs in common between the two fluoroquinolonesusceptible pools ( $\mathrm{S} 01$ and $\mathrm{S} 02$ ). The subtraction enriched for SNPs in 35 genes in common among fluoroquinolone-susceptible pools, DH10B, and REL606, but differed from SMS-3-5 (Figure 3b, genes listed in the red circle). Five of these genes are encoded on plasmid pSMS35_130 [GenBank: NG_010488.1], and the rest are on the chromosome. The identified EcSMS35labeled genes encode sequences found in other published sequences but none correspond to any well-annotated genes.

In agreement with our previously published data [20], the S83 allele of $\operatorname{gyr} A$ and the $\mathrm{S} 80$ allele of $\operatorname{par} C$ were found only in fluoroquinolone-susceptible pools. These susceptible $\operatorname{gyr} A$ and $\operatorname{par} C$ alleles were ubiquitous among the fluoroquinolone-susceptible $E$. coli genomes in GenBank and the Broad Institute. Potential alleles associated with antibiotic susceptibility may be as useful as their resistant counterparts in the identification of a resistance phenotype and will be an interesting area for future exploration.

\section{Additional Variants in Topoisomerase Genes Cluster in Pools}

In addition to the gyrAL83 variant, alleles of the gyrA gene (encoding amino acid Y/N87) and the parC gene (encoding I80 or $\mathrm{K} / \mathrm{G} 84$ ) occur frequently in fluoroquinolone-resistant $E$. coli $[10,20]$. Thus, we examined these alleles in the fluoroquinoloneresistant pools. Several pools contained the parCI80 variant as a mixed SNP. Although the gyrAY/N87- and parCK/G84-encoding alleles were found in the pools containing fluoroquinoloneresistant isolates at high frequency, none were unanimous in any of the pools. Pool M03 contained 13 isolates that are resistant only to fluoroquinolones (Table 1). The parCI80 allele was unanimous in this pool. Assuming that the mutation rate of the $\operatorname{par} C$ gene does not differ from other loci and that the likelihood of the parCS80 and parCI80 variants is equal, finding this unanimous SNP in a pool of 13 isolates $\left(1 / 2^{13}=1\right.$ in 8,192$)$ cannot be random chance. 

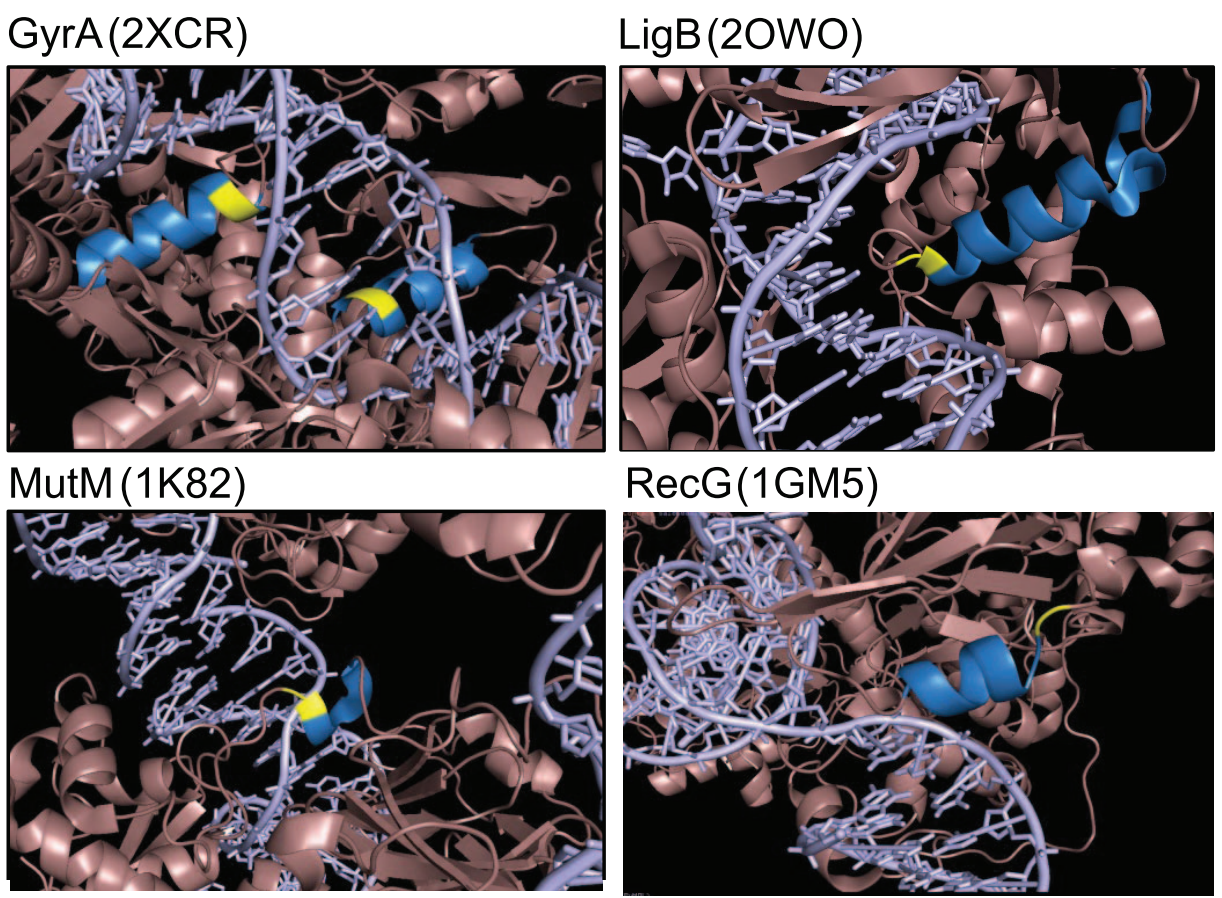

Figure 5. Position of variants in gyrase, LigB, MutM, and RecG co-crystal structures. The effect of amino acid substitutions was modeled using available co-crystal structures. GyrA PDB ID: 2XCR (from Staphylococcus aureus), LigA PDB ID: 20 WO (from E. coli), MutM PDB ID: 1K82 (from E. coli), and RecG PDB ID: 1GM5 (from Thermotoga maritima). Each amino acid variation (yellow), the $\alpha$-helix affected (blue), and DNA (purple) are shown. For gyrase and MutM, the variants associated with drug-susceptible laboratory E. coli strains are shown. For LigB and RecG the variants tightly linked to fluoroquinolone resistance are shown.

doi:10.1371/journal.pone.0065961.g005

This result may suggest that resistance to fluoroquinolones alone requires this $\operatorname{par} C$ variant. The linkage of the parCI80 with a pool resistant solely to fluoroquinolones implies that, in the absence of resistance mechanisms to other antibiotics, the addition of the parCI80 variant to the gyrAL83 variant may be necessary for clinically-relevant fluoroquinolone MICs. Experiments using isogenic strains showed that parCG84 only affected fluoroquinolone MICs when combined with both gyrAL83 and parCI80; parCK84 alone, in fact, decreased fluoroquinolone MICs [8]. In our clinical isolate collection [20] and as far as we know for any $E$. coli clinical isolate [12,45], parC mutations were never found alone.

\section{Cryptic Prophage Sequences in Clinical Isolates of E. coli}

Wang et al. found that deletion of any of three cryptic prophages (rac, e14, and CP4-6) lowered nalidixic acid MICs in the E. coli BW25113 K-12 strain [46]. Despite very good mappability and the very high coverage of the pooled sequences, these prophage sequences were detected infrequently (Figure S6a). Direct PCR screening for int $R$ for rac and per $R$ for CP-4-6 verified that these sequences were found equally in fluoroquinolone-susceptible and fluoroquinolone-resistant clinical isolates (Figure S6b). Thus, in spite of affecting quinolone MICs, these prophage sequences are not correlated with fluoroquinolone resistance in clinical isolates.

\section{Discussion}

Here, we report a novel GWAS analysis method using phenotype-based pooling, next generation sequencing, and SNP subtraction. The pooling approach, in addition to validating known variants (gyrA L83 and parC I80), can feasibly uncover genetic variants involved in a specific phenotype without the expense and challenge of sequencing and curating independent genome assemblies. Common mapping strategies assume that resequencing is done on descendant clones from the reference. We demonstrated that we can use indirectly related reference genomes as an annotation platform. While we used this method to identify novel alleles associated with fluoroquinolone resistance, we anticipate it could be used to probe for genomic commonalities associated with any phenotype.

The environment was implicated as a source of antibiotic resistance genes because of its diverse microbiota and the presence of antibiotics, both naturally produced and residual from agricultural use $[23,26]$. The antibiotic resistome of soil bacteria bears high nucleotide identity to the same resistance genes within known clinical pathogens, providing strong evidence of the relatedness between these two bacterial populations [47]. We uncovered extraordinary genomic similarity between SMS-3-5 and the multidrug-resistant E. coli clinical isolate pools that had the highest fluoroquinolone MICs (M05, M11, H02, and H03). The similarity was not confined to a specific region or regions, e.g., a pathogenicity island or a plasmid, but was evenly spread across the entire genome, with the highest nucleotide variation occurring at the regions adjacent to inversion and duplication events in the ancestry of the reference strains.

Fluoroquinolones kill bacteria through the accumulation of topoisomerase-bridged DNA breaks and through the release of oxidative radicals that damage the chromosome [42,48]. MutM removes mutagenic 8-oxoguanines in the genome after they have been incorporated as part of the bacterial GO system [49]. Deletion of MutM alone resulted in a modest increase in strain evolvability [50]. Deletion of the mut $M$ and mut $Y$ genes together increased E. coli survival in the presence of antibiotics [43], and demonstrated a hypermutator phenotype in Pseudomonas aeruginosa [51]. As a consequence of the inability to repair DNA mismatches, 
A

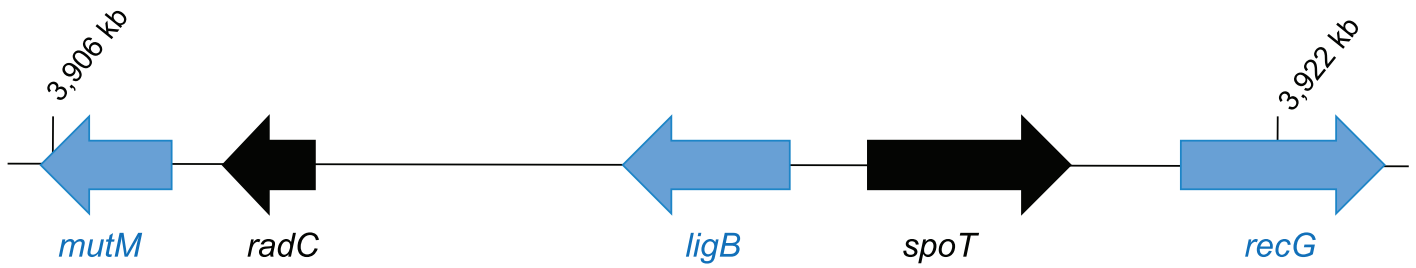

B

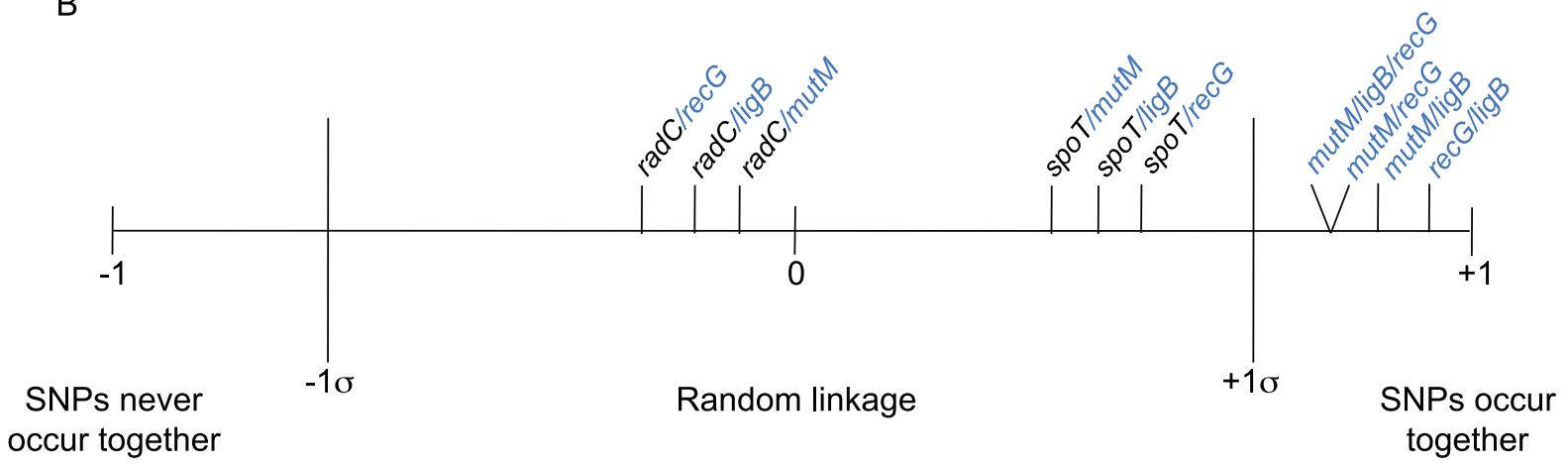

C

\begin{tabular}{|c|c|c|c|c|c|}
\hline Variant & mutM & $\mathrm{radc}$ & $\operatorname{lig} B$ & spot & recG \\
\hline mutM & N/A & $5.74 \mathrm{E}-1$ & $1.10 \mathrm{E}-15$ & $1.06 \mathrm{E}-5$ & $1.51 \mathrm{E}-12$ \\
\hline $\operatorname{rad} C$ & & $N / A$ & $7.31 \mathrm{E}-2$ & $1.87 \mathrm{E}-7$ & $8.25 \mathrm{E}-3$ \\
\hline $\operatorname{lig} B$ & & & N/A & $2.40 \mathrm{E}-8$ & $<2.2 \mathrm{E}-16$ \\
\hline spot & & & & N/A & $4.47 \mathrm{E}-10$ \\
\hline recG & & & & & $\mathrm{N} / \mathrm{A}$ \\
\hline
\end{tabular}

Figure 6. Linkage analysis of the $m u t M$, ligB, and recG fluoroquinolone resistance associated SNPs. A) Relative chromosomal location of mutM, radC, ligB, spoT, and recG in E. coli DH10B. B) To determine the linkage frequency, 39 complete E. coli genomes in GenBank and 83 draft genomes from the Broad Institute were analyzed at the positions of variants in mutM, ligB, and recG. Allelic variants in radC and spoT were selected for relative comparison based on their location between the genes of interest. The linkage of each pair of allelic variants was normalized to a range from never linked $(-1)$ to always linked $(+1)$. The sigma $(\sigma)$ denotes one standard deviation for a normal distribution. Strains with unannotated genes were not included in the analysis. C) The statistical significance of the linkage was calculated using two-by-two contingency tables and FONT NOT VALID ${ }^{2}$ analysis. With a Bonferroni correction for multiple comparisons, $p$-values $<0.005$ are considered significant. The statistical analysis was done using $\mathrm{R}$ version 2.12.2.

doi:10.1371/journal.pone.0065961.g006

bacteria accumulate genomic mutations. Clinically-isolated bacteria are frequently hypermutators. Though many of these mutations come at deleterious fitness costs, some mutations convey a selective advantage for occupying a niche within a host. Together with MutM, MutY prevents G:C to T:A transversions to protect the integrity of the chromosome [49]. The MutM variant we found, either alone or in combination with the LigB and RecG variants, may exhibit a hypermutator phenotype. If so, then perhaps the coordinated changes in these DNA modifying enzymes increase bacteria survival in response to the genotoxic stress created by fluoroquinolones. The mutation rate and evolvability of our identified SNP variants are topics of future research.

The modified residues of $\mathrm{rec} G$, mut $M$, and $\operatorname{lig} B$ each capped an alpha helix that is close in proximity to regions involved with DNA binding. The variants of RecG and LigB associated with fluoroquinolone resistance are predicted to stabilize the helices important for DNA binding. Stabilized forms of RecG and LigB may increase their ability to repair DNA damage induced by fluoroquinolones. Conversely, the MutM variant associated with fluoroquinolone resistance is predicted to destabilize the DNA binding helix, possibly by modifying nucleotide excision activity [43]. Taken together, these changes in $m u t M, \operatorname{lig} B$ and $\operatorname{rec} G$, could set the stage for the gyrA L83 encoding mutation to occur.
We uncovered alleles in genes that potentially represent the fluoroquinolone susceptibility phenotype. The concept of genetic variations associated with antibiotic susceptibility is distinct from the conventional view, where susceptibility is a default state upon which drug resistance variations are layered. By considering such a possibility, we found both known (such as those in $\operatorname{gyr} A$ and $\operatorname{par} C$ ) and novel alleles that can be linked to fluoroquinolone susceptibility. Maintaining drug susceptibility alleles in microflora could be an important way to combat the rise of drug resistance. The measure of frequency of alleles associated with antibiotic resistance or antibiotic susceptibility comprises a genomic signature that could be exploited in a diagnostic system to rapidly determine the antibiotic resistance of a pathogen. Such a system would minimize empirical antibiotic prescription, maximize treatment efficacy, and extend the useful life of the current antibiotic arsenal.

\section{Materials and Methods}

\section{Materials and Reagents}

Mueller-Hinton (MH) broth was from Difco (Sparks, MD); tryptone and yeast extract were from Becton Dickinson and Company (San Jose, CA); PureLink ${ }^{\mathrm{TM}}$ Pro 96 Genomic DNA Kit was from Invitrogen (Carlsbad, CA). NanoDrop ${ }^{\circledR}$ Spectrophotometer ND-1000 was from Thermo Scientific (Wilmington, DE). 


\section{Sequencing Pool Design}

Hosital-derived antibiotic susceptibility data ("S" for sensitive, "I" for intermediate, and "R" for resistant) for 17 antibiotics (listed in Table S1) and our quantitative susceptibility data for four fluoroquinolones (MICs for ciprofloxacin (CIP), gatifloxacin (GAT), levofloxacin (LVX), and norfloxacin(NOR)), were used to group 214 representative clinical isolates into pools by $k$-means clustering using Cluster 3.0 [29]. Strings of such data (e.g., R-S-I-R-R-S-40010 , etc) were used to represent each isolate in the $k$-means clustering. 32 pools were generated by the clustering algorithm. Following manual inspection, pools were removed if they contained only one strain or if they were highly similar to other pools, but lacked sufficient qualitative susceptibility data. Sixteen pools containing a total of 164 strains (Table 1) were submitted for whole-genome sequencing. The phenotypes represented in these pools ranged from susceptible to all drugs tested to nearly pan-drug resistant. We designated the pools as "M" when the isolates therein had fluoroquinolone MICs greater than the resistance breakpoints (ciprofloxacin $\geq 4 \mu \mathrm{g} / \mathrm{ml}$, gatifloxacin and levofloxacin $\geq 8 \mu \mathrm{g} / \mathrm{ml}$ and norfloxacin $\geq 16 \mu \mathrm{g} / \mathrm{ml}$ ) but less than $400 \mu \mathrm{g} / \mathrm{ml}$, and as " $\mathrm{H}$ " when fluoroquinolone MICs exceeded $400 \mu \mathrm{g} / \mathrm{ml}$.

\section{Genomic DNA Isolation and Pool Assembly}

Genomic DNA was isolated from each isolate using the PureLink $^{\text {TM }}$ Pro 96 Genomic DNA Kit and quantified using a NanoDrop ${ }^{\circledR}$ Spectrophotometer ND-1000. All DNA samples had $\mathrm{A}_{260} / \mathrm{A}_{280}$ greater than 1.8. Genomic DNA was pooled according to the results of the clustering analysis such that each genome was represented at equimolar concentrations within a pool.

\section{SOLiD ${ }^{\mathrm{TM}}$ Sequencing}

Pools of genomic DNA were sequenced using $2 \times 25$ bp matepaired libraries with the Applied Biosystems SOLiD ${ }^{\mathrm{TM}}$ System (Life Technologies). Briefly, 16-45 $\mu \mathrm{g}$ of DNA per library were sheared to $2.0 \mathrm{~kb}$ using the Covaris ${ }^{\mathrm{TM}} \mathrm{S} 2$ System according to the manufacturer's instructions. Genomic EcoP15I restriction enzyme sites were methylated prior to EcoP15I CAP Adaptor ligation. Samples were then size-selected and circularized incorporating the internal adaptor. In the subsequent EcoP15I restriction enzyme step, the DNA was cleaved 25-27 bp away from the unmethylated enzyme recognition site in the CAP adaptor forming the DNA mate-pair. Finally, P1 and P2 adaptors were ligated to the matepaired libraries for PCR amplification. Each library template was clonally amplified on SOLiD P1 beads using emulsion PCR. Templated ( $\mathrm{P} 2$ positive) beads were then enriched and deposited on an octet of a slide. SOLiD sequencing was carried out at $2 \times 25$ bp, using SOLiD v3.5 chemistry according to the manufacturer's instructions.

\section{Sequence Analysis}

Sequenced reads were mapped to selected genomes using BioScope v 1.2.1. $2 \times 25$ mate-pair reads were mapped to the reference genome allowing for up to two mismatches per read. The alignment with the highest mapping quality value was chosen as the primary alignment. If two or more alignments had the same score then one of them was randomly chosen as the primary alignment. Reads were then paired, allowing up to four mismatches across the pair by searching for pairs which are in the expected orientation and insert size (BioScope $v$ 1.2.1). SNP calling was performed using the BioScope diBayes algorithm, which incorporates position and probe errors as well as color quality value information for SNP calling, at medium-stringency setting (http://www3.appliedbiosystems.com/ cms/groups/mcb_marketing/documents/generaldocuments/ cms_057817.pdf). DiBayes filtered reads with mapping quality $<8$. DiBayes with medium stringency setting needs $2 x$ to call homozygous and $3 \mathrm{x}$ to call a heterozygous SNP. Annovar [52] was used to annotate the SNPs using gene lists from NCBI for the three platform genomes. Custom scripts were written in Perl for subsequent filtering of SNPs, and positions were further processed using Cluster [53], Java TreeView (http://jtreeview.sourceforge. net/), Sequencher (http://www.genecodes.com), and DataGraph (http://www.visualdatatools.com/DataGraph/). GO analysis was performed using the DAVID algorithm [54] and reference genome alignments were done on MUMmer [55].

\section{Sequence Comparison}

A secure HBase database housed all the SNP information and a custom Java program mined the collection of SNPs. This program sought SNPs that occurred only in either fluoroquinolonesusceptible or fluoroquinolone-resistant clinical isolates compared within each reference genome. A total of 709,163 unique SNPs from all the pools to reference comparisons were analyzed. Where potential fluoroquinolone-specific correlations did occur, the position and related information, including change, gene, pool identifier, and type of change were saved for downstream analysis.

\section{Atomic Structure Prediction}

Hydrogen atoms were placed on all polar heavy atoms in the crystal structures and their positions optimized using the modeling software Rosetta [56]. Stabilization interactions involving helixcapping motifs were identified if the corresponding residues established side chain/backbone hydrogen bonds of energies lower than $0.5 \mathrm{kcal} / \mathrm{mol}$.

\section{Overexpression Plasmid Construction}

Strains from the ASKA library [57] harboring plasmids containing the genes $r e c G$, $\operatorname{lig} B$, and $m u t M$ from MG1655 E. coli were obtained from the National Institute of Genetics, Shizuoka, Japan. Site directed mutagenesis of single amino acids was performed using the QuikChange protocol (Agilent) using the following primers. $\quad r e c G$ : 5'-CAATGAAAAATAGCGTGGGGACGGGCGGCGGTGTAC- ${ }^{\prime}$ and 5'-GTACACGGCGGCGCGTCGCCAGGCTATTTTTCATTG-3'. ligB: 5' CGCTTAGTCTGGCTGGGGTCAAAACAGGTTCTTGGG$3^{\prime}$ and 5' - CCGAAGAACGTGTTTTGACGCGAGCGAGACTAAGCG-3' . mutM: 5'-GGAAGGGCATAATGTGCTGGCGCATCTTGGACGGG-3' and 5'-CGGGTCGAAGATGGGGCAGGACATTATGCGCTTCG-3'. After point mutations were made and verified using Sanger sequencing, the expression plasmids containing each genomic variant were transformed separately into the corresponding Keio knockout mutant by standard methods.

\section{Measurement of MICs}

Ciprofloxacin MICs were determined using E-Test ${ }^{\circledR}$ strips (Biomérieux, Durham, NC). Measurements were made according to the manufacturer's instructions. Isogenic mutants were from the Keio knockout collection [41].

\section{$\mathrm{H}_{2} \mathrm{O}_{2}$ and UV Radiation Survival Assays}

Overnight cultures of the various deletion strains and those strains each complemented with the two different alleles were diluted 100-fold into $10 \mathrm{ml}$ of fresh Luria-Bertani (LB) medium containing $30 \mu \mathrm{g} / \mathrm{ml}$ (final concentration) chloramphenicol (Sigma, St. Louis, MO), when necessary. These cultures were incubated, with shaking, at $37^{\circ} \mathrm{C}$ to mid-logarithmic phase $\left(\mathrm{OD}_{600}=0.4\right)$. Cultures were then divided into two $5 \mathrm{ml}$ aliquots 
and $\mathrm{H}_{2} \mathrm{O}_{2}$ was added to a final concentration of $5 \mathrm{mM}$ to one aliquot. Samples were incubated for 20 minutes and treated with an excess $(50 \mu \mathrm{g} / \mathrm{ml})$ of catalase (Worthington Biochemical, Lakewood, NJ). Cultures were serially diluted, plated on LB agar, and incubated overnight at $37^{\circ} \mathrm{C}$. Colony forming units (CFUs) were enumerated after 16 hours. Each experiment was repeated at least three times.

For the UV irradiation experiments, cultures were grown as previously and serially diluted after reaching an $\mathrm{OD}_{600}=0.4$. A $100 \mu \mathrm{l}$ aliquot of the $10^{-5}$ dilution $\left(\sim 3 \times 10^{3}\right.$ cells $)$ was spread on LB agar. Plates were dried at room temperature for 20 minutes, exposed to the indicated doses of UV light using a CL-1000 ultraviolet crosslinker, and incubated overnight at $37^{\circ} \mathrm{C}$. CFUs were counted after 16 hours. Each experiment was repeated at least three times.

\section{Supporting Information}

Figure S1 Experimental design workflow. Selected isolates were clustered into pools based on their antibiotic resistance. Genomic DNA was pooled and sequenced on the SOLiD 3 platform. Sequence reads were mapped to using genomes DH10B (susceptible), REL606 (susceptible) and SMS-3-5 (multidrugresistant) as platforms. Unanimous SNP variants were called for each pool. For nonsynonymous alterations, the genes were identified. Genetic variation associated with fluoroquinolone resistance were those unanimous in all resistant pools, but not called in any fluoroquinolone-susceptible pools. Variations associated with fluoroquinolone susceptibility were those unanimous in the fluoroquinolone-susceptible pools, but not called in any fluoroquinolone-resistant pool.

(TIF)

Figure S2 SNP frequency plots relative to the DH10B genome. The frequency of unanimous SNPs in each pool was plotted along the $\mathrm{DH} 10 \mathrm{~B}$ chromosome. The $y$-axis for each pool was altered to allow visualization of SNPs and does not imply a value of frequency relative to other pools. Peaks in the plot represent regions of high variability for a pool. " $\mathrm{H}$ " pools are shown in red; "S" pools are in blue.

(EPS)

Figure S3 Genome-wide analysis of unanimous SNPs called relative to $\mathrm{DH} 10 \mathrm{~B}$. A) The percentage of genes containing variations associated with fluoroquinolone resistance. For each of the 13 fluoroquinolone-resistant pools, SNPs in genes were mapped to the DH10B genome. SNPs that were the same in identity and position were denoted as unanimous SNPs. SNPs that failed to match both of these criteria were named mixed SNPs. B) Occurrence of unanimous SNPs as a function of pool size. The total number of unanimous SNPs detected in each pool relative to $\mathrm{DH} 10 \mathrm{~B}$ was plotted versus pool size. Pool H01 was dropped from further analysis as an outlier by the Extreme Studentized Deviate test statistic. A best-fit line to the data for the remaining 13 resistant pools showed a weak negative correlation $\left(\mathrm{R}^{2}=0.0907\right)$, which was not statistically significant. C) Distribution of fluoroquinolone resistance-specific SNPs. SNPs found in any of the fluoroquinolone-susceptible pools were subtracted from the set. The distribution of the resistance-specific, unanimous SNPs is shown.

(EPS)

\section{References}

1. Mauldin PD, Salgado CD, Hansen IS, Durup DT, Bosso JA (2010) Attributable hospital cost and length of stay associated with health care-associated infections caused by antibiotic-resistant gram-negative bacteria. Antimicrob Agents Chemother 54: 109-115.
Figure S4 Phylogenetic analysis of published E. coli genomes. A phylogenetic analysis of the 33 E. coli genomes in GenBank showed SMS-3-5 (top row) is the most diverged. The heat map color range shows the relative number of additional sequence reads from each pool that could be mapped to each reference genome. (Blue $=$ fewest; Red $=$ most). A maximum number of additional sequence reads could be mapped to SMS-3-5.

(EPS)

Figure S5 Dotplots comparing SMS-3-5 to other E. coli genomes. A) Dotplot comparing the DH10B and W3110 genomes. The SMS-3-5 inversion is not present. B) Dotplot comparing SMS-3-5 and W3110. G-H) Dotplots comparing the SMS-3-5 genome to the genomes of two strains from each of the phylogenetic groups B2 $(\mathbf{G}, \mathbf{D}), \mathrm{D}(\mathbf{E}, \mathbf{F})$ and B1 $(\mathbf{G}, \mathbf{H})$. The inversion is found only in SMS-3-5 with the exception of IAI39 (E), which has a smaller inversion in the same region. All dotplots were made using Geneious software. Accession numbers are as follows: W3110 [GenBank: AP009048.1]; UTI89 [GenBank: CP000243]; NA114 [GenBank: CP002797]; IAI39 [GenBank: GU928164]; UMN026 [GenBank: CU928163]; SE11 [GenBank: AP009240]; and IAIl [GenBank: CU928160].

(EPS)

Figure S6 Representation of prophage in E. coli clinical isolates. A) Prophage coverage in pools. The short reads of each pool were probed for the presence of cryptic prophage. Coverage (y-axis) was normalized to the average coverage of $\mathrm{DH} 10 \mathrm{~B}$ core genes and displayed by intensity for each prophage in each pool (black, low coverage; red, high relative coverage). B) Presence of prophages in fluoroquinolone-susceptible and fluoroquinolone-resistant clinical isolates. Individual isolates were probed for the prophage genes int $R$ for rac and per $R$ for CP4-6 by PCR. The percentage of isolates testing positive for each gene is shown for all fluoroquinolonessusceptible $(n=18)$ and fluoroquinolone-resistant $(n=65)$ isolates tested. These isolates were selected to represent all pools shown in (A).

(EPS)

Table S1 Antibiotics classes.

(DOCX)

Table S2 Patient demographics and clinical isolate culture sites. (DOCX)

Table S3 Confirmation of allelic variants by Sanger sequencing. (DOCX)

\section{Acknowledgments}

We thank Dr. Daniel J. Catanese, Jr., Dr. Timothy Harkins, Dr. Martin M. Matzuk, and Kimberly Carlson-Banning for critically reading the manuscript; Dr. Christophe Herman for advice and reagents; and Huang Ting (Tim) Chang for technical assistance.

\section{Author Contributions}

Conceived and designed the experiments: MCS RS LZ. Performed the experiments: MCS MAE MJS CABC SS VS. Analyzed the data: MCS MAE TJB JREM PB VS SFM CCL DS LBB RS. Contributed reagents/ materials/analysis tools: MAE PB SFM CGL QD RJH DS LBB RS LZ. Wrote the paper: MCS MAE RS LZ TJB.

2. Boucher HW, Talbot GH, Bradley JS, Edwards JE, Gilbert D, et al. (2009) Bad bugs, no drugs: no ESKAPE! An update from the Infectious Diseases Society of America. Clin Infect Dis 48: 1-12. 
3. Klevens RM, Edwards JR, Richards CLJr, Horan TC, Gaynes RP, et al. (2007) Estimating health care-associated infections and deaths in U.S. hospitals, 2002. Public Health Rep 122: 160-166.

4. Peleg AY, Hooper DC (2010) Hospital-acquired infections due to gram-negative bacteria. N Engl J Med 362: 1804-1813.

5. Stone PW, Hedblom EC, Murphy DM, Miller SB (2005) The economic impact of infection control: making the business case for increased infection control resources. Am J Infect Control 33: 542-547.

6. Nikaido H (2009) Multidrug resistance in bacteria. Annu Rev Biochem 78: 119 146 .

7. Heisig P (1996) Genetic evidence for a role of parC mutations in development of high-level fluoroquinolone resistance in Escherichia coli. Antimicrob Agents Chemother 40: 879-885.

8. Morgan-Linnell SK, Zechiedrich L (2007) Contributions of the combined effects of topoisomerase mutations toward fluoroquinolone resistance in Escherichia coli. Antimicrob Agents Chemother 51: 4205-4208.

9. Singh R, Swick MC, Ledesma KR, Yang Z, Hu M, et al. (2012) Temporal interplay between efflux pumps and target mutations in development of antibiotic resistance in Escherichia coli. Antimicrob Agents Chemother 56: 16801685 .

10. Turner AK, Nair S, Wain J (2006) The acquisition of full fluoroquinolone resistance in Salmonella Typhi by accumulation of point mutations in the topoisomerase targets. J Antimicrob Chemother 58: 733-740.

11. Everett MJ, Jin YF, Ricci V, Piddock LJ (1996) Contributions of individual mechanisms to fluoroquinolone resistance in 36 Escherichia coli strains isolated from humans and animals. Antimicrob Agents Chemother 40: 2380-2386.

12. Ruiz J (2003) Mechanisms of resistance to quinolones: target alterations, decreased accumulation and DNA gyrase protection. J Antimicrob Chemother 51: 1109-1117.

13. Yoshida H, Bogaki M, Nakamura M, Nakamura S (1990) Quinolone resistancedetermining region in the DNA gyrase gyrA gene of Escherichia coli. Antimicrob Agents Chemother 34: 1271-1272.

14. Martinez-Martinez L, Pascual A, Jacoby GA (1998) Quinolone resistance from a transferable plasmid. Lancet 351: 797-799.

15. Robicsek A, Strahilevitz J, Jacoby GA, Macielag M, Abbanat D, et al. (2006) Fluoroquinolone-modifying enzyme: a new adaptation of a common aminoglycoside acetyltransferase. Nat Med 12: 83-88.

16. Tran JH, Jacoby GA (2002) Mechanism of plasmid-mediated quinolone resistance. Proc Natl Acad Sci U S A 99: 5638-5642.

17. Wang M, Tran JH, Jacoby GA, Zhang Y, Wang F, et al. (2003) Plasmidmediated quinolone resistance in clinical isolates of Escherichia coli from Shanghai, China. Antimicrob Agents Chemother 47: 2242-2248.

18. Robicsek A, Jacoby GA, Hooper DC (2006) The worldwide emergence of plasmid-mediated quinolone resistance. Lancet Infect Dis 6: 629-640.

19. Mazzariol A, Tokue Y, Kanegawa TM, Cornaglia G, Nikaido H (2000) Highlevel fluoroquinolone-resistant clinical isolates of Escherichia coli overproduce multidrug efflux protein AcrA. Antimicrob Agents Chemother 44: 3441-3443.

20. Becnel Boyd L, Maynard MJ, Morgan-Linnell SK, Horton LB, Sucgang R, et al. (2009) Relationships among ciprofloxacin, gatifloxacin, levofloxacin, and norfloxacin MICs for fluoroquinolone-resistant Escherichia coli clinical isolates. Antimicrob Agents Chemother 53: 229-234.

21. Swick MC, Morgan-Linnell SK, Carlson KM, Zechiedrich L (2011) Expression of multidrug efflux pump genes acrAB-tolC, mdfA, and norE in Escherichia coli clinical isolates as a function of fluoroquinolone and multidrug resistance. Antimicrob Agents Chemother 55: 921-924.

22. Bush WS, Moore JH (2012) Chapter 11: genome-wide association studies. PLoS Comput Biol 8: e1002822.

23. D'Costa VM, McGrann KM, Hughes DW, Wright GD (2006) Sampling the antibiotic resistome. Science 311: 374-377.

24. Wright GD (2007) The antibiotic resistome: the nexus of chemical and genetic diversity. Nat Rev Microbiol 5: 175-186.

25. Croucher NJ, Harris SR, Fraser C, Quail MA, Burton J, et al. (2011) Rapid pneumococcal evolution in response to clinical interventions. Science 331: 430434 .

26. Davies J, Davies D (2010) Origins and evolution of antibiotic resistance. Microbiol Mol Biol Rev 74: 417-433.

27. Fricke WF, Wright MS, Lindell AH, Harkins DM, Baker-Austin C, et al. (2008) Insights into the environmental resistance gene pool from the genome sequence of the multidrug-resistant environmental isolate Escherichia coli SMS-3-5. J Bacteriol 190: 6779-6794.

28. Boyd LB, Atmar RL, Randall GL, Hamill RJ, Steffen D, et al. (2008) Increased fluoroquinolone resistance with time in Escherichia coli from $>17,000$ patients at a large county hospital as a function of culture site, age, sex, and location. BMC Infect Dis 8: 4.

29. Eisen MB, Spellman PT, Brown PO, Botstein D (1998) Cluster analysis and display of genome-wide expression patterns. Proc Natl Acad Sci U S A 95: 14863-14868.
30. Kaas RS, Friis C, Ussery DW, Aarestrup FM (2012) Estimating variation within the genes and inferring the phylogeny of 186 sequenced diverse Escherichia coli genomes. BMC Genomics 13: 577.

31. Durfee T, Nelson R, Baldwin S, Plunkett G 3rd, Burland V, et al. (2008) The complete genome sequence of Escherichia coli DH10B: insights into the biology of a laboratory workhorse. J Bacteriol 190: 2597-2606.

32. Jeong H, Barbe V, Lee CH, Vallenet D, Yu DS, et al. (2009) Genome sequences of Escherichia coli B strains REL606 and BL21(DE3). J Mol Biol 394: 644-652.

33. Barrick JE, Yu DS, Yoon SH, Jeong H, Oh TK, et al. (2009) Genome evolution and adaptation in a long-term experiment with Escherichia coli. Nature 461: 1243-1247.

34. Daegelen P, Studier FW, Lenski RE, Cure S, Kim JF (2009) Tracing ancestors and relatives of Escherichia coli B, and the derivation of B strains REL606 and BL21(DE3). J Mol Biol 394: 634-643.

35. Sahl JW, Steinsland H, Redman JC, Angiuoli SV, Nataro JP, et al. (2011) A comparative genomic analysis of diverse clonal types of enterotoxigenic Escherichia coli reveals pathovar-specific conservation. Infect Immun 79: 950-960.

36. Keller I, Bensasson D, Nichols RA (2007) Transition-transversion bias is not universal: a counter example from grasshopper pseudogenes. PLoS Genet 3: $\mathrm{e} 22$.

37. Zhang Z, Gerstein M (2003) Patterns of nucleotide substitution, insertion and deletion in the human genome inferred from pseudogenes. Nucleic Acids Res 31: 5338-5348.

38. Coculescu BI (2009) Antimicrobial resistance induced by genetic changes. J Med Life 2: 114-123

39. Sutherland JH, Tse-Dinh YC (2010) Analysis of RuvABC and RecG involvement in the Escherichia coli response to the covalent topoisomerase-DNA complex. J Bacteriol 192: 4445-4451.

40. Rudolph CJ, Upton AL, Harris L, Lloyd RG (2009) Pathological replication in cells lacking RecG DNA translocase. Mol Microbiol 73: 352-366.

41. Baba T, Ara T, Hasegawa M, Takai Y, Okumura Y, et al. (2006) Construction of Escherichia coli K-12 in-frame, single-gene knockout mutants: the Keio collection. Mol Syst Biol 2: 20060008.

42. Dwyer DJ, Kohanski MA, Hayete B, Collins JJ (2007) Gyrase inhibitors induce an oxidative damage cellular death pathway in Escherichia coli. Mol Syst Biol 3: 91.

43. Foti JJ, Devadoss B, Winkler JA, Collins JJ, Walker GC (2012) Oxidation of the guanine nucleotide pool underlies cell death by bactericidal antibiotics. Science 336: $315-319$

44. Sriskanda V, Shuman S (2001) A second NAD(+)-dependent DNA ligase (LigB) in Escherichia coli. Nucleic Acids Res 29: 4930-4934.

45. Lu T, Zhao X, Drlica K (1999) Gatifloxacin activity against quinolone-resistant gyrase: allele-specific enhancement of bacteriostatic and bactericidal activities by the C-8-methoxy group. Antimicrob Agents Chemother 43: 2969-2974.

46. Wang X, Kim Y, Ma Q Hong SH, Pokusaeva K, et al. (2010) Cryptic prophages help bacteria cope with adverse environments. Nat Commun 1: 147.

47. Forsberg KJ, Reyes A, Wang B, Selleck EM, Sommer MO, et al. (2012) The shared antibiotic resistome of soil bacteria and human pathogens. Science 337: 1107-1111.

48. Kohanski MA, Dwyer DJ, Hayete B, Lawrence CA, Collins IJ (2007) A common mechanism of cellular death induced by bactericidal antibiotics. Cell 130: 797810.

49. Michaels ML, Miller JH (1992) The GO system protects organisms from the mutagenic effect of the spontaneous lesion 8-hydroxyguanine (7,8-dihydro-8oxoguanine). J Bacteriol 174: 6321-6325.

50. Orlen H, Hughes D (2006) Weak mutators can drive the evolution of fluoroquinolone resistance in Escherichia coli. Antimicrob Agents Chemother 50: 3454-3456.

51. Mandsberg LF, Macia MD, Bergmann KR, Christiansen LE, Alhede M, et al. (2011) Development of antibiotic resistance and up-regulation of the antimutator gene $p f p I$ in mutator Pseudomonas aeruginosa due to inactivation of two DNA oxidative repair genes (mut , mutM). FEMS Microbiol Lett 324: 28-37.

52. Wang K, Li M, Hakonarson H (2010) ANNOVAR: functional annotation of genetic variants from high-throughput sequencing data. Nucleic Acids Res 38: e164.

53. de Hoon MJ, Imoto S, Nolan J, Miyano S (2004) Open source clustering software. Bioinformatics 20: 1453-1454.

54. Huang da W, Sherman BT, Lempicki RA (2009) Systematic and integrative analysis of large gene lists using DAVID bioinformatics resources. Nat Protoc 4: 44-57.

55. Kurtz S, Phillippy A, Delcher AL, Smoot M, Shumway M, et al. (2004) Versatile and open software for comparing large genomes. Genome Biol 5: R12.

56. Das R, Baker D (2008) Macromolecular modeling with rosetta. Annu Rev Biochem 77: 363-382.

57. Kitagawa M, Ara T, Arifuzzaman M, Ioka-Nakamichi T, Inamoto E, et al (2005) Complete set of ORF clones of Escherichia coli ASKA library (a complete set of E. coli K-12 ORF archive): unique resources for biological research. DNA Res 12: 291-299. 